\title{
REDUCE-ing the link between smoking and prostate cancer
}

Lean smokers have an increased risk of developing high-grade prostate cancer, with delays in diagnosis further accentuating the association, according to evaluation of data taken from the REDUCE study.

The specific risks regarding prostate cancer and smoking are not clear. Previous

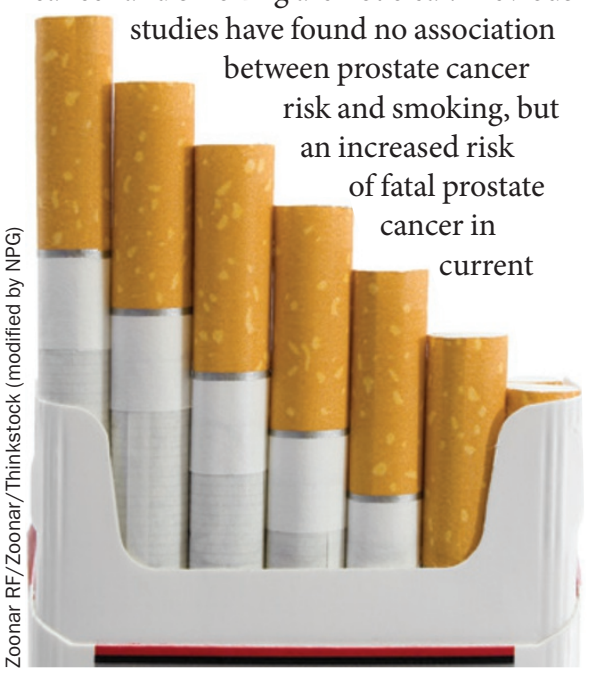

smokers compared with nonsmokers, which increased further in those who smoked the most cigarettes per day. One possibility for this discrepancy is that smokers often delay diagnosis, reducing their chances of successful prostate cancer treatment; another is that smoking affects aggressive disease to a greater degree than indolent disease. A recently published paper has now investigated this association further using data from the REDUCE trial.

Of the 6,240 patients included in the study, $45.8 \%$ never smoked, $14.5 \%$ were current smokers and $39.8 \%$ were former smokers. After adjusting for patient demographics and disease characteristics, current smoking was significantly associated with a decreased risk of receiving at least one on-study biopsy. Of those patients who did receive one biopsy without detection of prostate cancer, current smokers were $36 \%$ less likely to receive a second on-study biopsy.

In line with previous studies, the risk of prostate cancer diagnosis was unaffected by smoking status and no link was found with low-grade disease. However, current smokers had a $44 \%$ increased risk of having high-grade disease (OR 1.44, $P=0.028$ ) detected at their first on-study biopsy, an association not seen in former smokers. The researchers noted an association between smoking and obesity, so went on to further stratify patients by BMI. They observed that smoking was only associated with high-grade disease in men with BMI $<25 \mathrm{~kg} / \mathrm{m}^{2}$. The reason for this association was unclear, but the authors speculate that it might be due to a higher concentration of carcinogens in the serum in leaner men.

These results lend weight to the argument that smokers are less compliant to follow-up, suggesting a role for delayed diagnosis and treatment in the potential link between prostate cancer and smoking.

Annette Fenner

Original article Ho, T. et al. Smoking and risk of low- and high-grade prostate cancer: Results from the REDUCE study. Clin. Cancer Res. doi:10.1158/1078-0432.CCR-13-2394 